Review Article

\title{
Involvement of Cathepsins in Innate and Adaptive Immune Responses in Periodontitis
}

\author{
Xu Yan, ${ }^{1}$ Zhou Wu $\mathbb{D}^{2,3}$ Biyao Wang ${ }^{D},{ }^{1}$ Tianhao Yu, ${ }^{1}$ Yue Hu $\mathbb{D},{ }^{1}$ Sijian Wang, ${ }^{4}$ \\ Chunfu Deng, ${ }^{3}$ Baohong Zhao, ${ }^{4}$ Hiroshi Nakanishi, ${ }^{5}$ and Xinwen Zhang $\oplus^{4}$ \\ ${ }^{1}$ The VIP Department, School and Hospital of Stomatology, China Medical University, \\ Liaoning Provincial Key Laboratory of Oral Diseases, Shenyang 110002, China \\ ${ }^{2}$ Department of Aging Science and Pharmacology, Faculty of Dental Science, Kyushu University, Fukuoka 812-8582, Japan \\ ${ }^{3}$ OBT Research Center, Faculty of Dental Science, Kyushu University, Fukuoka 812-8582, Japan \\ ${ }^{4}$ Center of Implant Dentistry, School and Hospital of Stomatology, China Medical University, \\ Liaoning Provincial Key Laboratory of Oral Diseases, Shenyang 110002, China \\ ${ }^{5}$ Department of Pharmacology, Faculty of Pharmacy, Yasuda Women's University, Hiroshima 731-0153, Japan
}

Correspondence should be addressed to Zhou Wu; zhouw@dent.kyushu-u.ac.jp and Xinwen Zhang; zhangxinwen@cmu.edu.cn

Received 9 January 2020; Revised 27 February 2020; Accepted 7 March 2020; Published 31 March 2020

Guest Editor: Young-Su Yi

Copyright ( $12020 \mathrm{Xu}$ Yan et al. This is an open access article distributed under the Creative Commons Attribution License, which permits unrestricted use, distribution, and reproduction in any medium, provided the original work is properly cited.

Periodontitis is an infectious disease whereby the chronic inflammatory process of the periodontium stimulated by bacterial products induces specific host cell responses. The activation of the host cell immune system upregulates the production of inflammatory mediators, comprising cytokines and proteolytic enzymes, which contribute to inflammation and bone destruction. It has been well known that periodontitis is related to systemic inflammation which links to numerous systemic diseases, including diabetes and arteriosclerosis. Furthermore, periodontitis has been reported in association with neurodegenerative diseases such as Alzheimer's disease (AD) in the brain. Regarding immune responses and inflammation, cathepsin B (CatB) plays pivotal role for the induction of IL-1 $\beta$, cathepsin K- (CatK-) dependent active toll-like receptor 9 (TLR9) signaling, and cathepsin S (CatS) which involves in regulating both TLR signaling and maturation of the MHC class II complex. Notably, both the production and proteolytic activities of cathepsins are upregulated in chronic inflammatory diseases, including periodontitis. In the present review, we focus on the roles of cathepsins in the innate and adaptive immune responses within periodontitis. We believe that understanding the roles of cathepsins in the immune responses in periodontitis would help to elucidate the therapeutic strategies of periodontitis, thus benefit for reduction of systemic diseases as well as neurodegenerative diseases in the global aging society.

\section{Introduction}

Immune responses, including the innate and adaptive immune responses, are components of a defensive mechanism which has become increasingly specialized with evolution. The human immune system is able to generate great quantities of specialized cells and molecules capable of recognizing and eliminating an apparently unlimited diversity of foreign invaders by functioning cooperatively [1]. Of note, although these specialized cells and molecules accumulate at inflammatory sites to efficiently remove invading agents, they may also amplify the inflammatory response and impair the surrounding tissues [2]. Therefore, under the inflammatory conditions, the inflammatory-responsive cell-related immune responses must be tightly controlled. Periodontitis is a chronic infectious disease whereby chronic inflammation of the periodontium involves interactions among bacterial products, numerous cell populations, and inflammatory mediators. The initiation of periodontitis might be attributed to dental plaques and complex and diverse microbial biofilms that form on the teeth. In particular, substances released from these biofilms, including lipopolysaccharides (LPS), antigens, and other virulence factors, gain access to the gingival tissues. As a 
result, the innate and adaptive immune responses are elicited, thus leading to the activation of host defense cells. Collectively, inflammatory mediators, which comprise cytokines and proteolytic enzymes, induce tissue destruction and bone resorption [3].

Cathepsin, a term derived from the Greek word kathepsein (meaning to digest), is a protease that is functionally active in a slightly acidic environment. There are 11 human cysteine cathepsin isoforms, referred to as $\mathrm{B}, \mathrm{C}, \mathrm{F}, \mathrm{H}$, $\mathrm{K}, \mathrm{L}, \mathrm{O}, \mathrm{S}, \mathrm{V}, \mathrm{X}$, and $\mathrm{W}$ [4]. Cathepsins are primarily intracellular enzymes responsible for nonspecific bulk proteolysis in the endosomal/lysosomal system, which degrades both intracellular and extracellular proteins [5]. However, cathepsins are involved in producing immune modulators by the limited proteolysis processing. For example, cathepsin $\mathrm{B}$ (CatB) involves in the production of interleukin-1 $\beta$ (IL$1 \beta)[6,7]$ and TNF- $\alpha[8]$ and cathepsin $\mathrm{K}(\mathrm{CatK})$ involves in toll-like receptor 9 (TLR9) activation [9]. In addition, cathepsin S (CatS) mediates TLR signaling transduction and regulates major histocompatibility complex (MHC) class IIdependent $\mathrm{CD}^{+}{ }^{+} \mathrm{T}$-cell activation [10]. Indeed, both the production and proteolytic activities of CatS are upregulated under conditions closely related to chronic inflammatory diseases, including periodontitis [11]. In the present review, we summarize the current knowledge on the involvement of cathepsins in regulating innate and adaptive immune responses in periodontitis. Given the roles of cathepsins in immune/inflammatory responses, the regulation of cathepsins will be helpful for the management of cellular immune responses in patients with periodontitis, and thus beneficial to prevent and relax the systemic diseases as well as neurodegenerative diseases in the global aging society.

\section{Innate and Adaptive Immune Responses in Periodontitis}

2.1. Periodontal Pathogens and TLRs. Periodontitis is caused by specific bacterial infection. The bacterial species living in polymicrobial biofilms or below the gingival margin proliferate largely as a result of the inflammation initiated by specific subgingival species. It is widely accepted that specific microorganisms are associated with specific periodontal diseases, and the search for the periodontal pathogens responsible for distinct periodontal conditions is under way $[12,13]$. The "red complex," which is composed of the pathogens Porphyromonas gingivalis, Tannerella forsythia, and Treponema denticola, has been shown to exist in the biofilms where progressive periodontitis is shown [14]. Bacterial components, such as LPS, peptidoglycans, lipoteichoic acids, proteases, and toxins, which stimulate the inflammatory activity, can be detected in the biofilms on the surface of the tooth [15]. Antigens and toxic products released by bacteria, such as LPS and peptidoglycans, are identified by TLRs on the surface of host cells and can trigger cellular signaling of host cells [16]. On the host side, the diverse inflammatory cell types and resident cells of the tissues respond to bacterial infection and induce cellular immune responses that are innate and adaptive immune responses [17]. TLRs can detect multiple pathogen-related molecular patterns, including LPS, bacterial lipoproteins, lipoteichoic acids, flagellin, CpGDNA of bacteria and viruses, double-stranded RNA, and single-stranded viral RNA [18]. To date, 11 different TLRs (TLR1-11) have been identified [16]. It has been known that lipopolysaccharides from Porphyromonas gingivalis ( $P$. gingivalis LPS) is recognized by TLR2/TLR4 $[19,20]$ while flagellin in Tannerella forsythia is recognized by TLR5 [21]. The TLR pathway is crucial in the immune responses in periodontitis because TLRs are bound to their corresponding antigens, which triggers intracellular immune responses to produce inflammatory-related mediators [22].

2.2. Macrophages in Periodontitis. Macrophages are myeloid cells of hematopoietic origin, which play important role in the local immune responses [23]. The major functions of macrophages include elimination of invading bacteria, recruitment of immune cells to the site of infection, production of cytokines and chemokines, and activation of the adaptive immune response through TLR signaling [22]. The functions of activated macrophages are regulated by mediators produced by $\mathrm{T}$ cells. The classically activated macrophages (M1) are regulated by interferon (IFN) $-\gamma$ and LPS [24], while alternatively activated macrophages (M2) are produced in response to IL-4 or IL-13 [25]. M2 macrophages have been shown to play a role in relief of inflammation and thus have decreased capacity to produce cytokines. Macrophages express high levels of TLR2 and TLR4 [26], which shift to the M1 phenotype in gingival tissues of patients with chronic periodontitis [27]. Interestingly, M1 macrophages can transform into M2 macrophages by exosomes derived from gingival mesenchymal stem cells, which is beneficial for suppressing the immune responses [28].

Accumulating studies have demonstrated that macrophage serves as an important route for local immune responses in periodontitis because macrophage can produce multiple proinflammatory mediators, including tumor necrosis factor (TNF- $\alpha$ ) and IL- $1 \beta$ and IL- 6 through activating the nuclear factor kappa-light-chain-enhancer of activated $B$ $(\mathrm{NF}-\kappa \mathrm{B})$ pathway [29]. In addition to the contribution to innate immune response, TNF- $\alpha$ also serves as a significant route for alveolar bone resorption in periodontitis for inducing the differentiation and activation of osteoclasts [30]. IL- $1 \beta$ activates generation of matrix metalloproteinase (MMP)-9 in periodontitis [31] and promotes the expression of the receptor activator of nuclear factor kappa-B ligand (RANKL), contributing to osteoclastogenesis in periodontitis $[32,33]$. A recent report has shown that IL- $1 \beta$-expressed inflammatory macrophages produce amyloid $\beta$ (the main component of the hall marker in the brain of AD patients) in the gingival tissues of the patients with periodontitis as well as in the liver of mice after chronic systemic $P$. gingivalis infection, indicating that inflammatory macrophages in periodontitis may contribute to neurodegenerative diseases such as AD [34]. IL-6 triggers the generation of vascular endothelial growth factor (VEGF) and MMP-1, making great contribution to the development of periodontitis [35] and synergic effects of IL- $1 \beta$ and IL- 6 upregulate MMPs, 
contributing to the tissue destruction in periodontitis by collagen degradation and bone resorption [36]. It is noted that chemical ablation of macrophages in mice prevents the $P$. gingivalis-induced alveolar bone resorption, demonstrating important roles for macrophages during periodontitis [37].

\subsection{Dendritic Cells in Periodontitis. Dendritic cells (DCs) are} located adjacent to the epithelia of blood vessels and the mucosa that shield soft tissues from microbial invasion. Bacteria or antigen-antibody complexes of bacteria or bacterial products that breach the epithelia and mucosa will encounter DCs and induce DC responses. DCs are typically the first immune cells to encounter and respond to invading microorganisms [38]. The nature of this DC response is essential in determining the type of acquired immune response that is induced. Adaptive immune response relies on the formation of appropriate peptides from foreign proteins and the consequent presentation on MHC I or II complexes [39], and DCs are able to initiate the elimination of the MHC II-associated chaperone invariant chain (Ii) from MHC II $[40,41]$. Upon the stimulation by pathogens, such as $P$. gingivalis, antigen presentation by DCs leads to the activation and subsequent differentiation of $\mathrm{CD} 4^{+} \mathrm{T}$ cells [36]. The unique immune-stimulatory activity of DC stems from their ability to efficiently capture and present antigens and optimally express cytokines after $P$. gingivalis LPS stimulation [42]. Naïve DCs are found in large numbers in gingival tissues, and DCs are matured in the inflamed gingival tissues from patients with periodontitis [43] which particularly serve as immune stimulators [44]. Considering the roles of DCs for cytokine production and differentiation of $\mathrm{CD} 4^{+} \mathrm{T}$ cells, DCs are reckoned to be the bridge between the innate and adaptive immune responses in periodontitis.

2.4. T Cells in Cellular Immune Responses. T cells can be activated in response to oral bacterial antigens [45], and $\mathrm{CD}^{+} \mathrm{T}$ cells play key roles in the development of a mouse model of periodontitis [46]. $\mathrm{CD}^{+}$T-cell-dependent responses are initiated by the recognition of MHC class II peptide complexes on antigen-presenting cells (APCs), such as DCs and macrophages. During the process, the invariant chain is sequentially degraded to leupeptin-induced peptide 10 (lip10) and ultimately to class II-associated Ii peptide (CLIP), which remains in the peptide-binding groove $[47,48]$. Then, CLIP is removed from the class II molecules catalyzed by the human leukocyte antigen DM which is capable of binding with antigenic peptides. Subsequently, the CLIP complex is transported to the cell surface, on which the antigen is presented to cognate $\mathrm{T}$ cells, initiating an immune response [49]. The naïve $\mathrm{CD}^{+} \mathrm{T}$ helper (Th0) cells can differentiate into $\mathrm{CD}^{+}$cell subtypes, including Th1, Th2, Th17, and regulatory T cells (Tregs), depending on the cytokines which are produced. In the presence of IL-12, Th1 cells are derived by IFN- $\gamma$ and TNF- $\alpha$, while Th 2 cells are derived in the presence of IL-4, which produce IL-4, IL-5, and IL-10. Th17 cells, which secrete IL-17, IL-23, and IL-22, are derived in the presence of TGF- $\beta$, IL- $1 \beta$, and IL- 6 . In contrast, Tregs are raised in the presence of TGF- $\beta$, which secretes the immunosuppressive cytokines IL-10 and TGF- $\beta$. In the participation of immune responses, IL-17 stimulates the production of inflammatory mediators, including TNF$\alpha$, IL-6, and IL- $1 \beta$, while Tregs effectively regulate the resolution of inflammation $[50,51]$. An in vitro study has reported that $\mathrm{CD} 4^{+} \mathrm{T}$ cells with its proinflammatory cytokines IFN- $\gamma$ and IL- 6 serve as important routes for alveolar bone resorption in mice infected with $P$. gingivalis [46]. Tregs and Th17 cells have been demonstrated in periodontal tissues which are involved in periodontal disease processes [52]. Th17 cells promote bone destruction [51, 53], while Tregs protect alveolar bone by inhibiting osteoclastogenesis [54]. Therefore, the plasticity and cross-talk in T-cell subsets are vital for the regulation of the cellular immune response during periodontitis and therapeutic strategies comprising Tregs inhibiting the immuno-inflammatory response and restoring alveolar bone homeostasis [55]. Indeed, the effect of antibiotic therapy in regulating Treg-Th17 plasticity in humans with periodontitis is demonstrated [56].

\section{Involvement of Cathepsin B in Innate Immune Responses}

3.1. CatB in Periodontitis. CatB, functioning as an endopeptidase at neutral $\mathrm{pH}$, is also found in the extralysosomal sites involving the cytosol, plasma membrane, and pericellular spaces [57]. In the noninvasive diagnostic body fluid, gingival crevicular fluid (GCF), CatB was detected mainly in macrophages when monocytes were migrating into the gingival crevice $[58,59]$. The protease activity of CatB in GCF is closely associated with the GCF volume and the severity of periodontitis $[60,61]$. CatB in GCF plays a major role in the pathology of periodontitis with respect to connective tissue breakdown and bone resorption. It is demonstrated that there is an imbalance between cathepsin B and the endogenous inhibitor cystatin $\mathrm{C}$, with the elevated level of cathepsin B and a decreased level of cystatin C [62]. As a result of the proteinases from $P$. gingivalis infection, the hemostasis of CatB activity is disrupted, contributing to the destruction in periodontitis [63]. Therefore, the activity and the amount of cathepsin B and cystatin C in GCF can potentially serve as a predictor of attachment loss and an indicator of the progression of the disease $[61,64,65]$. CatB plays a significant role in promoting chronic inflammation in periodontitis. It is reported that $\mathrm{CatB}$ regulates the expression of collagens III and IV by fibroblasts in response to a TLR2 agonist, $P$. gingivalis LPS [66]. Moreover, CatB recently has been determined to be involved in the production of amyloid $\beta$, the main component of the hall marker in the brain of $\mathrm{AD}$ patients, in the macrophages in the gingival tissues of the patients with periodontitis and in the liver of mice after chronic systemic $P$. gingivalis infection [67].

3.2. CatB in $I L-1 \beta$ Processing. IL- $1 \beta$ is recognized as the master mediator in innate immune responses, and two signals are required for IL- $1 \beta$ processing. One is NF- $\kappa \mathrm{B}$ - 
dependent pro-IL- $1 \beta$ production, and the other is caspase-1dependent proteolytic processing of pro-IL- $1 \beta$ to mature IL$1 \beta$. CatB plays a critical role in both signals of IL- $1 \beta$ processing because CatB involves in NF- $\kappa \mathrm{B}$ activation [68] and activation of caspase-1 $[6,7]$. We have demonstrated that CatB was colocalized with caspase-1, and treatment with CA-074Me, a specific CatB inhibitor, markedly inhibited caspase- 1 expression, resulting in a decreased production of IL-1 $\beta$ [69-71]. These findings are consistent with the observations that IL- $1 \beta$ and CatB are colocalized in phagolysosomes, and that the secretion of IL- $1 \beta$ is through the exocytosis of phagolysosomes in LPS-activated human monocytes [72]. The CatB expression is upregulated in the macrophages which involves the production of IL-1 $\beta$ and amyloid $\beta$ in gingival tissues of the patients with periodontitis $[67,73]$. Furthermore, CatB indirectly involves in the activation of caspase-1 through the proteolytic maturation of caspase-11 [74].

3.3. CatB in TNF- $\alpha$ Trafficking. Membrane-associated (m) TNF- $\alpha$ is a type II transmembrane precursor which is delivered from the trans-Golgi network to the recycling endosome $[75,76]$. Membrane-associated TNF- $\alpha$ is transported to the cell surface, where it is cleaved by the TNF$\alpha$-converting enzyme [77]. Membrane fusion among the TNF- $\alpha$-containing vesicles from the trans-Golgi network, the recycling endosomes, and the cell surface membrane is mediated by the interactions among various trans-soluble$\mathrm{N}$-ethylmaleimide-sensitive factor-attachment protein SNAP receptor (SNARE) family members [78]. Recent studies have revealed that the accumulation of newly synthesized mTNF- $\alpha$ originally occurs in the Golgi complex [79]. The mTNF-containing vesicles are then translocated from the trans-Golgi network to the recycling endosome and subsequently to the cell surface through two different membrane fusion processes $[75,76]$. The first fusion process is mediated by Q-SNAREs, including syntaxin 6 , syntaxin 7 , and vesicle transport, through interacting with t-SNARE homolog $1 \mathrm{~b}$ (Vit1b) of the Golgi complex TNF- $\alpha$ carrier vesicle and the R-SNARE vesicle-associated membrane protein 3 (VAMP3) of the recycling endosome. The second fusion process is mediated by the interactions between VAMP3 of the recycling endosome and the Q-SNARE complex which consists of syntaxin 4 and SNAP-23. LPS triggers expression of the Q-SNARE components, including syntaxin 4 and SNAP-23 for the accommodation of increased trafficking during TNF- $\alpha$ secretion in macrophages [80].

A recent report shows that cytosolic CatB is imperative for fusion of the TNF- $\alpha$-containing vesicles to the plasma membrane [8]. CatB can be localized in the nucleus and downregulate transcriptions [81]. However, there is no difference in the levels of syntaxin 4 and SNAP-23 in the CA074Me-treated or CatB-/- macrophages, suggesting that CatB does not downregulate the SNARE components. There is a possibility that CatB has functions in regulating TNF- $\alpha$ vesicle trafficking through regulation of the SNARE components, either at the transcriptional or posttranslational levels in THP-1 and primary human monocytic cells considering that CatB was reported to be involved in both transcription and posttranslational protein processes, such as protein [82] and thyroglobulin [83]. A new role for intracellular CatB activity involved in TNF- $\alpha$ signaling is suggested in the report [8].

Therefore, CatB plays a critical role in regulating innate immune responses in periodontitis by controlling production of IL- $1 \beta$ and TNF- $\alpha$.

\section{Involvement of Cathepsin $S$ in Adaptive Immune Responses}

4.1. CatS in Periodontitis. The general role of CatS is breaking down antigenic and antimicrobial peptides, involving antigen processing and presentation [84-86]. In addition, CatS also serves as an elastase, destroying extracellular matrix proteins, comprising collagen and proteins of the bacterial outer membrane $[84,87]$. Cat $S$ is generated by immune cells, including macrophages and DCs $[88,89]$. In an analysis of healthy buccal gingival tissues from Rhesus monkeys, the transcription of CatS was altered in aged healthy tissues compared with younger animals. These agerelated immune pathways were associated with periodontal health [90]. CatS was also found to be upregulated in rats with experimental periodontitis and human patients with periodontitis [11,91]. CatS is recognized as one of the hub proteins in the protein-protein interaction network of 726 differentially expressed genes in periodontitis and plays an essential role in bone loss involved in periodontitis progression [58, 91].

4.2. CatS in MHC II Antigen Presenting and $C D 4^{+}$T-Cell Activation. CatS is essential in MHC II antigen processing and presentation $[92,93]$. Indeed, CatS null mice show a considerable variation in generation of MHC II-bound Ii fragments, and presentation due to the fact that the Ii degradation in professional APCs is substantially diminished, where CatS is abundantly expressed [94, 95]. In addition, endocytosis selectively targets exogenous material to CatS in human DCs [96]. Enrichment of MHC II molecules within late endocytic structures has consistently been seen in splenic DCs of CatS-deficient mice [97]. Pharmacological or genetic inhibition of CatS results in defective TLR2 signaling in the $P$. gingivalis LPS-exposed DCs, indicating CatS may be involved in innate immune responses [10, 42].

4.3. CatS in Promoting of Th1 and Th17. Cathepsin S, which is predominantly generated by monocytes/macrophages and DCs, is involved in the ultimate proteolytic cleavage stage of the invariant chain in APCs during TLR2 signaling $[48,85,88]$. DCs induce the differentiation of naive $\mathrm{CD} 4^{+}$ T cells into various subpopulations, comprising Th1 (IFN- $\gamma$ ) or Th17 (Th17) $[98,99]$. Recently, we reported that CatS is crucial for the differentiation of naïve $\mathrm{CD} 4^{+} \mathrm{T}$ cells [10]. We have found that CatS deficiency significantly relieves lower Th1 cell responses, accompanied with a decreased level of IFN- $\gamma$ [10]. Moreover, we have demonstrated that CatS 


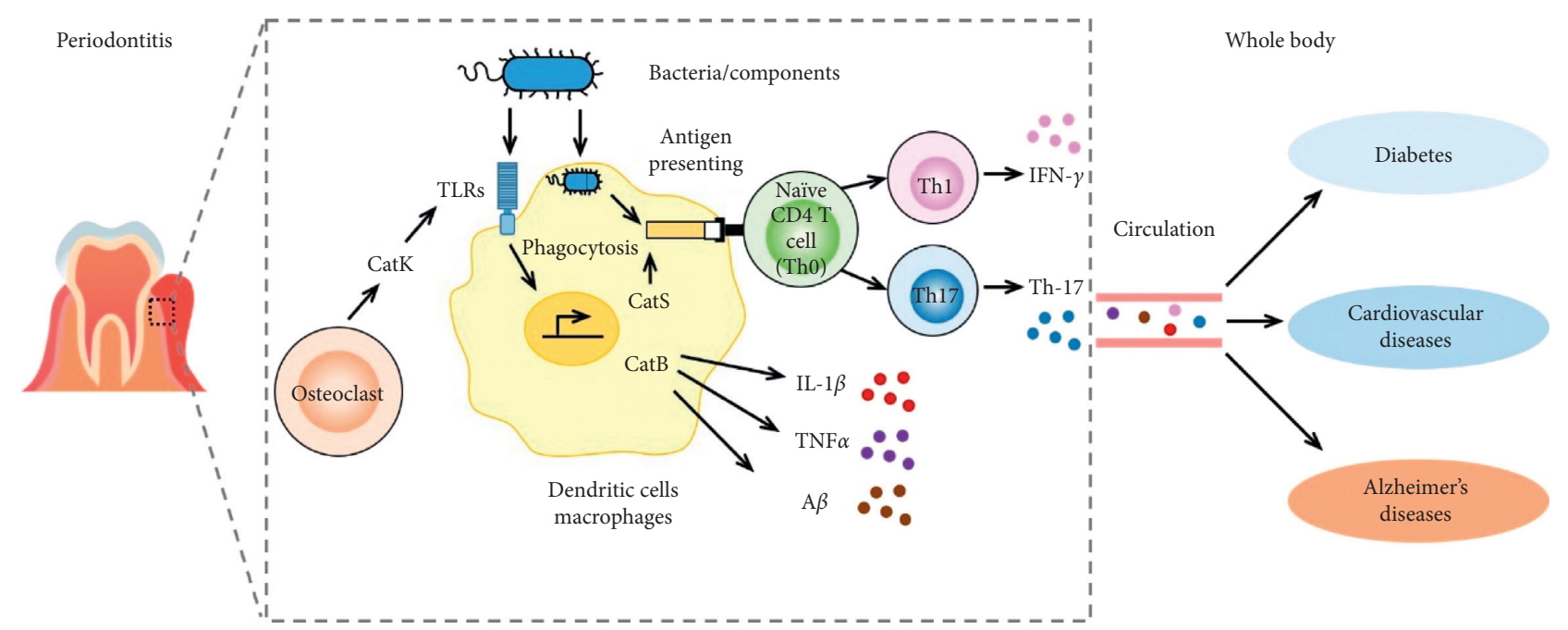

FIGURE 1: A schematic illustration of the cathepsin-related immune responses in periodontitis linking to the diseases within the whole body. In the bacteria (components)-stimulated cells, cathepsin B involves in activation of TLR signaling to produce IL- $1 \beta$, TNF- $\alpha$, and amyloid (A) $\beta$, and cathepsin S involves in maturation of MHC class II for driving CD4 ${ }^{+}$(helper) T cells to produce IFN- $\gamma$ (Th1) and IL-17 (Th17). Cathepsin $\mathrm{K}$ involves in activation of the TLR/autophagy pathway to produce type I interferon (IFN). The periodontitis cathepsin-related proinflammatory mediators involve in systemic diseases and Alzheimer's disease via the circulation.

promotes differentiation of Th17 cells in response to $P$. gingivalis LPS-exposed DCs through protease-activated receptor (PAR) 2-dependent IL-6 production [42]. Subsequently, the increased IL-6 triggers the differentiation of Th17 cells, illustrating the significant role of CatS in the differentiation of Th17 cells, after the exposure to P. gingivalis LPS [42]. Considering the critical roles of CatS in adaptive immune responses through promoting the differentiation of both Th1 and Th17 cells in periodontitis, CatS may provide a novel therapeutic target for treatment of periodontitis.

\section{Involvement of Cathepsin $\mathrm{K}$ in Immune Responses}

5.1. CatK in Periodontitis. Cathepsin $\mathrm{K}$ (CatK) is dominantly generated by osteoclasts, which serves as an important regulator for bone resorption [100]. CatK is essential for normal bone resorption via degrading collagens and gelatin, the latter being a hydrolysis product of collagen $[101,102]$. And it also dissolves type I collagen, the major component of the organic bone matrix [103]. Recently, several studies have demonstrated that CatK plays a crucial role in alveolar bone resorption in patients with periodontitis. It is reported that there are elevated levels of CatK in GCF of patients with periodontitis and periimplantitis [104-107]. Once the hemostasis is destroyed, bone resorption will occur in periodontitis. In patients with chronic periodontitis, CatK levels were greatly elevated in smokers compared to nonsmokers, indicating a positive influence of smoking on CatK [108]. Moreover, CatK in GCF is not only derived from osteoclasts, but also from fibroblasts, macrophages, and gingival epithelial cells, contributing to the attachment loss and alveolar bone resorption [109]. Pharmacologic or genetic inhibition of CatK results in defective TLR9 signaling in DCs in response to unmethylated CpGDNA, without affecting antigen-presenting ability [110].

5.2. CatK in Autophagy. The elevated level of CatK not only directly induces bone resorption, but also makes impact on innate immune indirectly contributing to periodontitis and even autoimmune diseases, especially rheumatoid arthritis (RA) [9]. As the level of CatK is elevated, there would be an activated TLR9 signaling in DCs and macrophages and consequently, osteoclastic bone resorption $[103,110]$. The CpGDNA can be recognized by TLR9, which can trigger autophagy [111-113]. In response to the activation of TLR9, the autophagy protein microtubule-associated protein $1 \mathrm{~A} /$ $1 \mathrm{~B}$-light chain 3 (LC3) aggregates $\mathrm{I} \kappa \mathrm{B}$ kinase $\alpha(\mathrm{IKK} \alpha)$ for type I interferon generation [112]. Moreover, there is a significant increase in the TLR9 downstream proteins (IKK $\beta$ and MYD88) and TFEB, which are greatly correlated with autophagy, in response to the activation of TLR9 $[114,115]$. Coinciding with the conclusion that the activation of TLR9 can induce the downstream autophagy pathway, the inhibition of CatK can suppress this response via downregulating the expression of TLR9 $[9,116]$. Therefore, CatK may involve in innate immune responses in periodontitis through the TLR/autophagy pathway.

\section{Conclusion}

The involvement of cathepsins in the immune responses of periodontitis contribute to systemic diseases, including diabetes, cardiovascular diseases, and neurodegenerative disease, including $\mathrm{AD}$, especially increasing in aged population (Figure 1). We believe that regulation of cathepsins, including CatB, CatS, and CatK, in cellular immune responses in patients with periodontitis will be beneficial for 
reduction of systemic diseases and neurodegenerative diseases in the global aging society.

\section{Conflicts of Interest}

The authors declare no conflicts of interest.

\section{Acknowledgments}

This work was financially supported by grants from the National Natural Science Foundation of China (no. 81700977 and 81500858), the Technology Project Program of Liaoning Province of China (no. 20170541059) from the Nature Sciences Foundation of Liaoning Province of China, and the Japanese KAKENHI Grant no. 16K11478 (grants-inaid for scientific research to Z.W.). The authors would like to thank the Department of Aging Science and Pharmacology, Faculty of Dental Science, Kyushu University, Fukuoka, Japan, for their support and allowing them to use their facilities.

\section{References}

[1] D. M. Clancy, G. P. Sullivan, H. B. T. Moran et al., "Extracellular neutrophil proteases are efficient regulators of IL1, IL-33, and IL-36 cytokine activity but poor effectors of microbial killing," Cell Reports, vol. 22, no. 11, pp. 29372950, 2018.

[2] S. J. Weiss, "Tissue destruction by neutrophils," The New England Journal of Medicine, vol. 320, no. 6, pp. 365-376, 1989.

[3] E. M. Cardoso, C. Reis, and M. C. Manzanares-Céspedes, "Chronic periodontitis, inflammatory cytokines, and interrelationship with other chronic diseases," Postgraduate Medicine, vol. 130, no. 1, pp. 98-104, 2018.

[4] B. Turk, D. Turk, and V. Turk, "Lysosomal cysteine proteases: more than scavengers," Biochimica et Biophysica Acta, vol. 1477, no. 1-2, pp. 98-111, 2000.

[5] V. Turk, B. Turk, and D. Turk, "New embo members' review: lysosomal cysteine proteases: facts and opportunities," The EMBO Journal, vol. 20, no. 17, pp. 4629-4633, 2001.

[6] R. I. Campden and Y. Zhang, "The role of lysosomal cysteine cathepsins in NLRP3 inflammasome activation," Archives of Biochemistry and Biophysics, vol. 670, pp. 32-42, 2019.

[7] Y. Zhang, Y. Chen, Y. Zhang, P.-L. Li, and X. Li, "Contribution of cathepsin B-dependent Nlrp3 inflammasome activation to nicotine-induced endothelial barrier dysfunction," European Journal of Pharmacology, vol. 865, p. 172795, 2019.

[8] S.-D. Ha, A. Martins, K. Khazaie, J. Han, B. M. Chan, and S. O. Kim, "Cathepsin B is involved in the trafficking of TNFalpha-containing vesicles to the plasma membrane in macrophages," The Journal of Immunology, vol. 181, no. 1, pp. 690-697, 2008.

[9] W. Wei, J. Ren, W. Yin et al., "Inhibition of Ctsk modulates periodontitis with arthritis via downregulation of TLR9 and autophagy," Cell Proliferation, vol. 53, no. 1, Article ID e12722, 2020.

[10] X. Zhang, Z. Wu, Y. Hayashi, R. Okada, and H. Nakanishi, "Peripheral role of cathepsin S in Th1 cell-dependent transition of nerve injury-induced acute pain to a chronic pain state," Journal of Neuroscience, vol. 34, no. 8, pp. 3013-3022, 2014.
[11] S. Memmert, A. Damanaki, A. V. B. Nogueira et al., "Role of cathepsin S in periodontal inflammation and infection," Mediators of Inflammation, vol. 2017, Article ID 4786170, 10 pages, 2017.

[12] G. A. Weijden, M. F. Timmerman, E. Reijerse, G. N. Wolffe, A. J. Winkelhoff, and U. Velden, "The prevalence of $A$. actinomycetemcomitans, $P$. gingivalis and $P$. intermedia in selected subjects with periodontitis," Journal of Clinical Periodontology, vol. 21, no. 9, pp. 583-588, 1994.

[13] A. Mombelli, "Microbiological monitoring," Journal of Clinical Periodontology, vol. 23, no. 3, pp. 251-257, 1996.

[14] S. Schulz, M. Porsch, I. Grosse, K. Hoffmann, H.-G. Schaller, and S. Reichert, "Comparison of the oral microbiome of patients with generalized aggressive periodontitis and periodontitis-free subjects," Archives of Oral Biology, vol. 99, pp. 169-176, 2019.

[15] T. R. Fitzsimmons, S. Ge, and P. M. Bartold, "Compromised inflammatory cytokine response to $P$. gingivalis LPS by fibroblasts from inflamed human gingiva," Clinical Oral Investigations, vol. 22, no. 2, pp. 919-927, 2018.

[16] K. Takeda and S. Akira, “Toll-like receptors," Current Protocols in Immunology, vol. 109, no. 1, 2015.

[17] T. E. Van Dyke and A. J. van Winkelhoff, "Infection and inflammatory mechanisms," Journal of Clinical Periodontology, vol. 40, no. 14, pp. S1-S7, 2013.

[18] T. Kawai and S. Akira, "The role of pattern-recognition receptors in innate immunity: update on toll-like receptors," Nature Immunology, vol. 11, no. 5, pp. 373-384, 2010.

[19] K. Q. de Andrade, C. L. C. Almeida-da-Silva, and R. Coutinho-Silva, "Immunological pathways triggered by Porphyromonas gingivalis and Fusobacterium nucleatum: therapeutic possibilities?" Mediators of Inflammation, vol. 2019, Article ID 7241312, 20 pages, 2019.

[20] T. Maekawa, J. L. Krauss, T. Abe et al., "Porphyromonas gingivalis manipulates complement and TLR signaling to uncouple bacterial clearance from inflammation and promote dysbiosis," Cell Host \& Microbe, vol. 15, no. 6, pp. 768-778, 2014.

[21] M. R. Benakanakere, Q. Li, M. A. Eskan et al., "Modulation of TLR2 protein expression by miR-105 in human oral keratinocytes," Journal of Biological Chemistry, vol. 284, no. 34, pp. 23107-23115, 2009.

[22] B. Song, Y. Zhang, L. Chen et al., "The role of toll-like receptors in periodontitis," Oral Diseases, vol. 23, no. 2, pp. 168-180, 2017.

[23] C. Varol, A. Mildner, and S. Jung, "Macrophages: development and tissue specialization," Annual Review of Immunology, vol. 33, no. 1, pp. 643-675, 2015.

[24] C. M. Minutti, B. García-Fojeda, A. Sáenz et al., "Surfactant protein A prevents IFN- $\gamma /$ IFN- $\gamma$ receptor interaction and attenuates classical activation of human alveolar macrophages," The Journal of Immunology, vol. 197, no. 2, pp. 590-598, 2016.

[25] D. R. Herbert, B. Douglas, and K. Zullo, "Group 2 innate lymphoid cells (ILC2): type 2 immunity and helminth immunity," International Journal of Molecular Sciences, vol. 20, no. 9, 2019.

[26] H. AlQallaf, Y. Hamada, S. Blanchard, D. Shin, R. Gregory, and M. Srinivasan, "Differential profiles of soluble and cellular toll like receptor (TLR)-2 and 4 in chronic periodontitis," PLoS One, vol. 13, no. 12, Article ID e0200231, 2018. 
[27] J. Yang, Y. Zhu, D. Duan et al., "Enhanced activity of macrophage M1/M2 phenotypes in periodontitis," Archives of Oral Biology, vol. 96, pp. 234-242, 2018.

[28] R. Wang, Q. Ji, C. Meng et al., "Role of gingival mesenchymal stem cell exosomes in macrophage polarization under inflammatory conditions," International Immunopharmacology, no. 81, Article ID 106030, 2019.

[29] Y. Xiao, C. Li, M. Gu et al., "Protein disulfide isomerase silence inhibits inflammatory functions of macrophages by suppressing reactive oxygen species and NF- $\kappa \mathrm{B}$ pathway," Inflammation, vol. 41, no. 2, pp. 614-625, 2018.

[30] T. Tenkumo, L. Rojas-Sanchez, J. R. Vanegas Saenz et al., "Reduction of inflammation in a chronic periodontitis model in rats by TNF-alpha gene silencing with a topically applied siRNA-loaded calcium phosphate paste," Acta Biomaterialia, vol. 105, pp. 263-279, 2020.

[31] R. Cheng, Z. Wu, M. Li, M. Shao, and T. Hu, "Interleukinlbeta is a potential therapeutic target for periodontitis: a narrative review," International Journal of Oral Science, vol. 12 , no. 1 , p. 2, 2020.

[32] G. N. Belibasakis and N. Bostanci, "The RANKL-OPG system in clinical periodontology," Journal of Clinical Periodontology, vol. 39, no. 3, pp. 239-248, 2012.

[33] N. C.-N. Huynh, V. Everts, P. Pavasant, and R. S. Ampornaramveth, "Interleukin- $1 \beta$ induces human cementoblasts to support osteoclastogenesis," International Journal of Oral Science, vol. 9, no. 12, p. e5, 2017.

[34] C. Fu, Z. Wei, and D. Zhang, "PTEN inhibits inflammatory bone loss in ligature-induced periodontitis via IL1 and TNFalpha," BioMed Research International, vol. 2019, Article ID 6712591, 9 pages, 2019.

[35] K. Naruishi and T. Nagata, "Biological effects of interleukin6 on Gingival Fibroblasts: cytokine regulation in periodontitis," Journal of Cellular Physiology, vol. 233, no. 9, pp. 6393-6400, 2018.

[36] J. L. Hor, P. G. Whitney, A. Zaid, A. G. Brooks, W. R. Heath, and S. N. Mueller, "Spatiotemporally distinct interactions with dendritic cell subsets facilitates $\mathrm{CD} 4^{+}$and $\mathrm{CD} 8^{+} \mathrm{T}$ cell activation to localized viral infection," Immunity, vol. 43, no. 3, pp. 554-565, 2015.

[37] R. S. Lam, N. M. O’Brien-Simpson, J. C. Lenzo et al., "Macrophage depletion abates Porphyromonas gingivalisinduced alveolar bone resorption in mice," Journal of Immunology, vol. 193, no. 5, pp. 2349-2362, 2014.

[38] S. C. Eisenbarth, "Dendritic cell subsets in T cell programming: location dictates function," Nature Reviews Immunology, vol. 19, no. 2, pp. 89-103, 2019.

[39] T. Zavasnik-Bergant and B. Turk, "Cysteine proteases: destruction ability versus immunomodulation capacity in immune cells," Biological Chemistry, vol. 388, no. 11, pp. 1141-1149, 2007.

[40] K. Honey and A. Y. Rudensky, "Lysosomal cysteine proteases regulate antigen presentation," Nature Reviews Immunology, vol. 3, no. 6, pp. 472-482, 2003.

[41] R. D. A. Wilkinson, R. Williams, C. J. Scott, and R. E. Burden, "Cathepsin S: therapeutic, diagnostic, and prognostic potential," Biological Chemistry, vol. 396, no. 8, pp. 867-882, 2015.

[42] M. Dekita, Z. Wu, J. Ni et al., "Cathepsin S is involved in Th17 differentiation through the upregulation of IL- 6 by activating PAR-2 after systemic exposure to lipopolysaccharide from Porphyromonas gingivalis," Frontiers in Pharmacology, vol. 8, p. 470, 2017.
[43] J. Carrion, E. Scisci, B. Miles et al., "Microbial carriage state of peripheral blood dendritic cells (DCs) in chronic periodontitis influences DC differentiation, atherogenic potential," The Journal of Immunology, vol. 189, no. 6, pp. 3178-3187, 2012.

[44] G. R. Souto, C. M. Queiroz-Junior, M. H. de Abreu, F. O. Costa, and R. A. Mesquita, "Pro-inflammatory, Th1, Th2, Th17 cytokines and dendritic cells: a cross-sectional study in chronic periodontitis," PLoS One, vol. 9, no. 3, Article ID e91636, 2014.

[45] N. R. Bennett, D. B. Zwick, A. H. Courtney, and L. L. Kiessling, "Multivalent antigens for promoting B and T cell activation," ACS Chemical Biology, vol. 10, no. 8, pp. 1817-1824, 2015.

[46] P. J. Baker, M. Dixon, R. T. Evans, L. Dufour, E. Johnson, and D. C. Roopenian, "CD4 ${ }^{+} \mathrm{T}$ cells and the proinflammatory cytokines gamma interferon and interleukin- 6 contribute to alveolar bone loss in mice," Infection and Immunity, vol. 67, no. 6, pp. 2804-2809, 1999.

[47] T. Y. Nakagawa, W. H. Brissette, P. D. Lira et al., "Impaired invariant chain degradation and antigen presentation and diminished collagen-induced arthritis in cathepsin S null mice," Immunity, vol. 10, no. 2, pp. 207-217, 1999.

[48] R. J. Riese, G.-P. Shi, J. Villadangos et al., "Regulation of CD1 function and NK1.1+ T cell selection and maturation by cathepsin S," Immunity, vol. 15, no. 6, pp. 909-919, 2001.

[49] J. A. Villadangos, R. J. Riese, C. Peters, H. A. Chapman, and H. L. Ploegh, "Degradation of mouse invariant chain: roles of cathepsins S and D and the influence of major histocompatibility complex polymorphism," Journal of Experimental Medicine, vol. 186, no. 4, pp. 549-560, 1997.

[50] N. Gagliani, M. C. A. Vesely, A. Iseppon et al., "Th17 cells transdifferentiate into regulatory $\mathrm{T}$ cells during resolution of inflammation," Nature, vol. 523, no. 7559, pp. 221-225, 2015.

[51] T. Okui, Y. Aoki, H. Ito, T. Honda, and K. Yamazaki, "The presence of $\mathrm{IL}_{-1} 17^{+} / \mathrm{FOXP}^{+}$double-positive cells in periodontitis," Journal of Dental Research, vol. 91, no. 6, pp. 574-579, 2012.

[52] V. P. B. Parachuru, D. E. Coates, T. J. Milne, H. M. Hussaini, A. M. Rich, and G. J. Seymour, "Forkhead box P3-positive regulatory T-cells and interleukin 17-positive T-helper 17 cells in chronic inflammatory periodontal disease," Journal of Periodontal Research, vol. 49, no. 6, pp. 817-826, 2014.

[53] K. Bunte and T. Beikler, "Th17 cells and the IL-23/IL-17 Axis in the pathogenesis of periodontitis and immune-mediated inflammatory diseases," International Journal of Molecular Sciences, vol. 20, no. 14, 2019.

[54] Y. K. Han, Y. Jin, Y. B. Miao, T. Shi, and X. P. Lin, "CD8 Foxp $^{+} \mathrm{T}$ cells affect alveolar bone homeostasis via modulating Tregs/Th17 during induced periodontitis: an adoptive transfer experiment," Inflammation, vol. 41, no. 5, pp. 1791-1803, 2018.

[55] E. A. Cafferata, A. Jerez, R. Vernal, G. Monasterio, N. Pandis, and C. M. Faggion Jr., "The therapeutic potential of regulatory T lymphocytes in periodontitis: a systematic review," Journal of Periodontal Research, vol. 54, no. 3, pp. 207-217, 2019.

[56] M. Rajendran, S. Looney, N. Singh et al., "Systemic antibiotic therapy reduces circulating inflammatory dendritic cells and Treg-Th17 plasticity in periodontitis," The Journal of Immunology, vol. 202, no. 9, pp. 2690-2699, 2019.

[57] H. Hentze, X. Y. Lin, M. S. K. Choi, and A. G. Porter, "Critical role for cathepsin B in mediating caspase-1-dependent interleukin-18 maturation and caspase-1- 
independent necrosis triggered by the microbial toxin nigericin," Cell Death \& Differentiation, vol. 10, no. 9, pp. 956-968, 2003.

[58] B. Zhao, M. Takami, A. Yamada et al., "Interferon regulatory factor-8 regulates bone metabolism by suppressing osteoclastogenesis," Nature Medicine, vol. 15, no. 9, pp. 1066-1071, 2009.

[59] S. W. Cox, E. M. Rodriguez-Gonzalez, V. Booth, and B. M. Eley, "Secretory leukocyte protease inhibitor and its potential interactions with elastase and cathepsin B in gingival crevicular fluid and saliva from patients with chronic periodontitis," Journal of Periodontal Research, vol. 41, no. 5, pp. 477-485, 2006.

[60] K. Kunimatsu, K. Yamamoto, E. Ichimaru, Y. Kato, and I. Kato, "Cathepsins B, H and L activities in gingival crevicular fluid from chronic adult periodontitis patients and experimental gingivitis subjects," Journal of Periodontal Research, vol. 25, no. 2, pp. 69-73, 1990.

[61] E. Ichimaru, M. Tanoue, M. Tani et al., "Cathepsin B in gingival crevicular fluid of adult periodontitis patients: identification by immunological and enzymological methods," Inflammation Research, vol. 45, no. 6, pp. 277282, 1996.

[62] R. Elkaim, S. Werner, L. Kocgozlu, and H. Tenenbaum, "P. gingivalis regulates the expression of cathepsin $\mathrm{B}$ and cystatin C," Journal of Dental Research, vol. 87, no. 10, pp. 932-936, 2008.

[63] M. Abrahamson, M. Wikstrom, J. Potempa, S. Renvert, and A. Hall, "Modification of cystatin C activity by bacterial proteinases and neutrophil elastase in periodontitis," Molecular Pathology, vol. 50, no. 6, pp. 291-297, 1997.

[64] B. M. Eley and S. W. Cox, "The relationship between gingival crevicular fluid cathepsin B activity and periodontal attachment loss in chronic periodontitis patients: a 2-year longitudinal study," Journal of Periodontal Research, vol. 31, no. 6, pp. 381-392, 1996.

[65] H. Y. Chen, S. W. Cox, and B. M. Eley, "Cathepsin B, alpha2macroglobulin and cystatin levels in gingival crevicular fluid from chronic periodontitis patients," Journal of Clinical Periodontology, vol. 25, no. 1, pp. 34-41, 1998.

[66] X. Li, Z. Wu, J. Ni et al., "Cathepsin B regulates collagen expression by fibroblasts via prolonging TLR2/NF-kappaB activation," Oxidative Medicine and Cellular Longevity, vol. 2016, Article ID 7894247, 12 pages, 2016.

[67] R. Nie, Z. Wu, J. Ni et al., "Porphyromonas gingivalis infection induces amyloid- $\beta$ accumulation in monocytes/ macrophages," Journal of Alzheimer's Disease, vol. 72, no. 2, pp. 479-494, 2019.

[68] J. Ni, Z. Wu, C. Peterts, K. Yamamoto, H. Qing, and H. Nakanishi, "The critical role of proteolytic relay through cathepsins B and E in the phenotypic change of microglia/ macrophage," Journal of Neuroscience, vol. 35, no. 36, pp. 12488-12501, 2015.

[69] Z. Wu, L. Sun, S. Hashioka et al., "Differential pathways for interleukin- $1 \beta$ production activated by chromogranin $\mathrm{A}$ and amyloid $\beta$ in microglia," Neurobiology of Aging, vol. 34, no. 12, pp. 2715-2725, 2013.

[70] K. Terada, J. Yamada, Y. Hayashi et al., "Involvement of cathepsin $\mathrm{B}$ in the processing and secretion of interleukin- $1 \beta$ in chromogranin A-stimulated microglia," Glia, vol. 58, no. 1, pp. 114-124, 2010.

[71] L. Sun, Z. Wu, Y. Hayashi et al., "Microglial cathepsin B contributes to the initiation of peripheral inflammation- induced chronic pain," Journal of Neuroscience, vol. 32, no. 33, pp. 11330-11342, 2012.

[72] C. Andrei, C. Dazzi, L. Lotti, M. R. Torrisi, G. Chimini, and A. Rubartelli, "The secretory route of the leaderless protein interleukin $1 \beta$ involves exocytosis of endolysosome-related vesicles," Molecular Biology of the Cell, vol. 10, no. 5, pp. 1463-1475, 1999.

[73] Y. Liu, Z. Wu, X. Zhang et al., "Leptomeningeal cells transduce peripheral macrophages inflammatory signal to microglia in reponse to Porphyromonas gingivalis LPS," Mediators of Inflammation, vol. 2013, Article ID 407562, 11 pages, 2013.

[74] S.-J. Kang, S. Wang, H. Hara et al., "Dual role of caspase-11 in mediating activation of caspase- 1 and caspase- 3 under pathological conditions," Journal of Cell Biology, vol. 149, no. 3, pp. 613-622, 2000.

[75] R. Z. Murray, J. G. Kay, D. G. Sangermani, and J. L. Stow, "A role for the phagosome in cytokine secretion," Science, vol. 310, no. 5753, pp. 1492-1495, 2005.

[76] R. Z. Murray, F. G. Wylie, T. Khromykh, D. A. Hume, and J. L. Stow, "Syntaxin 6 and Vtilb form a novel SNARE complex, which is up-regulated in activated macrophages to facilitate exocytosis of tumor necrosis factor- $\alpha$," Journal of Biological Chemistry, vol. 280, no. 11, pp. 10478-10483, 2005.

[77] R. A. Black, C. T. Rauch, C. J. Kozlosky et al., “A metalloproteinase disintegrin that releases tumour-necrosis factor- $\alpha$ from cells," Nature, vol. 385, no. 6618, pp. 729-733, 1997.

[78] J. L. Stow, A. P. Manderson, and R. Z. Murray, "SNAREing immunity: the role of SNAREs in the immune system," Nature Reviews Immunology, vol. 6, no. 12, pp. 919-929, 2006.

[79] W. Shurety, A. Merino-Trigo, D. Brown, D. A. Hume, and J. L. Stow, "Localization and post-Golgi trafficking of tumor necrosis factor-alpha in macrophages," Journal of Interferon \& Cytokine Research, vol. 20, no. 4, pp. 427-438, 2000.

[80] J. K. Pagan, F. G. Wylie, S. Joseph et al., "The t-SNARE syntaxin 4 is regulated during macrophage activation to function in membrane traffic and cytokine secretion," Current Biology, vol. 13, no. 2, pp. 156-160, 2003.

[81] M. C. Pizzorno, "Nuclear cathepsin B-like protease cleaves transcription factor YY1 in differentiated cells," Biochimica et Biophysica Acta (BBA)-Molecular Basis of Disease, vol. 1536, no. 1, pp. 31-42, 2001.

[82] I. Jutras and T. L. Reudelhuber, "Prorenin processing by cathepsin B in vitro and in transfected cells," FEBS Letters, vol. 443, no. 1, pp. 48-52, 1999.

[83] A. D. Dunn and J. T. Dunn, "Cysteine proteinases from human thyroids and their actions on thyroglobulin," Endocrinology, vol. 123, no. 2, pp. 1089-1097, 1988.

[84] D. P. Dickinson, "Cysteine peptidases of mammals: their biological roles and potential effects in the oral cavity and other tissues in health and disease," Critical Reviews in Oral Biology and Medicine: An Official Publication of the American Association of Oral Biologists, vol. 13, no. 3, pp. 238-275, 2002.

[85] H. Nakanishi, "Microglial functions and proteases," Molecular Neurobiology, vol. 27, no. 2, pp. 163-176, 2003.

[86] P.-M. Andrault, S. A. Samsonov, G. Weber et al., "Antimicrobial peptide LL-37 is both a substrate of cathepsins S and $\mathrm{K}$ and a selective inhibitor of cathepsin L," Biochemistry, vol. 54, no. 17, pp. 2785-2798, 2015.

[87] P. Pietschmann, U. Foger-Samwald, W. Sipos, and M. Rauner, "The role of cathepsins in osteoimmunology," 
Critical Reviews in Eukaryotic Gene Expression, vol. 23, no. 1, pp. 11-26, 2013.

[88] S. Petanceska, P. Canoll, and L. A. Devi, "Expression of rat cathepsin S in phagocytic cells," Journal of Biological Chemistry, vol. 271, no. 8, pp. 4403-4409, 1996.

[89] V. Zavasnik-Bergant, A. Sekirnik, R. Golouh, V. Turk, and J. Kos, "Immunochemical localisation of cathepsin S, cathepsin L and MHC class II-associated p41 isoform of invariant chain in human lymph node tissue," Biological Chemistry, vol. 382, no. 5, pp. 799-804, 2001.

[90] O. A. Gonzalez, M. J. Novak, S. Kirakodu et al., "Comparative analysis of gingival tissue antigen presentation pathways in ageing and periodontitis," Journal of Clinical Periodontology, vol. 41, no. 4, pp. 327-339, 2014.

[91] L. Song, J. Yao, Z. He, and B. Xu, "Genes related to inflammation and bone loss process in periodontitis suggested by bioinformatics methods," BMC Oral Health, vol. 15, p. 105, 2015.

[92] T. Zavasnik-Bergant and B. Turk, "Cysteine cathepsins in the immune response," Tissue Antigens, vol. 67, no. 5, pp. 349355, 2006.

[93] Z. Meng, W. Klinngam, M. C. Edman, and S. F. HammAlvarez, "Interferon-gamma treatment in vitro elicits some of the changes in cathepsin $S$ and antigen presentation characteristic of lacrimal glands and corneas from the NOD mouse model of Sjogren's syndrome," PLoS One, vol. 12, no. 9, Article ID e0184781, 2017.

[94] G.-P. Shi, J. A. Villadangos, G. Dranoff et al., "Cathepsin S required for normal MHC class II peptide loading and germinal center development," Immunity, vol. 10, no. 2, pp. 197-206, 1999.

[95] J. Bania, E. Gatti, H. Lelouard et al., "Human cathepsin S, but not cathepsin L, degrades efficiently MHC class II-associated invariant chain in nonprofessional APCs," Proceedings of the National Academy of Sciences, vol. 100, no. 11, pp. 66646669, 2003

[96] M. Reich, P. F. van Swieten, V. Sommandas et al., "Endocytosis targets exogenous material selectively to cathepsin $S$ in live human dendritic cells, while cell-penetrating peptides mediate nonselective transport to cysteine cathepsins," Journal of Leukocyte Biology, vol. 81, no. 4, pp. 990-1001, 2007.

[97] C. Driessen, R. A. R. Bryant, A.-M. Lennon-Duménil et al., "Cathepsin S controls the trafficking and maturation of MHC class II molecules in dendritic cells," Journal of Cell Biology, vol. 147, no. 4, pp. 775-790, 1999.

[98] P. McGuirk and K. H. G. Mills, "Pathogen-specific regulatory T cells provoke a shift in the Th1/Th2 paradigm in immunity to infectious diseases," Trends in Immunology, vol. 23, no. 9, pp. $450-455,2002$.

[99] L. E. Harrington, R. D. Hatton, P. R. Mangan et al., "Interleukin 17-producing $\mathrm{CD} 4^{+}$effector T cells develop via a lineage distinct from the T helper type 1 and 2 lineages," Nature Immunology, vol. 6, no. 11, pp. 1123-1132, 2005.

[100] M. Novinec and B. Lenarčič, "Cathepsin K: a unique collagenolytic cysteine peptidase," Biological Chemistry, vol. 394, no. 9, pp. 1163-1179, 2013.

[101] M. Matsumoto, M. Kogawa, S. Wada et al., "Essential role of p38 mitogen-activated protein kinase in cathepsin $\mathrm{K}$ gene expression during osteoclastogenesis through association of NFATc1 and PU.1," Journal of Biological Chemistry, vol. 279, no. 44, pp. 45969-45979, 2004.

[102] A. H. Aguda, P. Panwar, X. Du, N. T. Nguyen, G. D. Brayer, and D. Brömme, "Structural basis of collagen fiber degradation by cathepsin K," Proceedings of the National Academy of Sciences, vol. 111, no. 49, pp. 17474-17479, 2014.

[103] B. R. Troen, "The regulation of cathepsin K gene expression," Annals of the New York Academy of Sciences, vol. 1068, no. 1, pp. 165-172, 2006.

[104] L. Funkelstein, M. Beinfeld, A. Minokadeh, J. Zadina, and V. Hook, "Unique biological function of cathepsin L in secretory vesicles for biosynthesis of neuropeptides," $\mathrm{Neu}$ ropeptides, vol. 44, no. 6, pp. 457-466, 2010.

[105] G. Garg, A. R. Pradeep, and M. K. Thorat, "Effect of nonsurgical periodontal therapy on crevicular fluid levels of cathepsin K in periodontitis," Archives of Oral Biology, vol. 54, no. 11, pp. 1046-1051, 2009.

[106] N. Yamalik, S. Gunday, K. Kilinc, E. Karabulut, E. Berker, and T. F. Tozum, "Analysis of cathepsin-K levels in biologic fluids from healthy or diseased natural teeth and dental implants," The International Journal of Oral \& Maxillofacial Implants, vol. 26, no. 5, pp. 991-997, 2011.

[107] N. Yamalik, S. Günday, S. Uysal, K. Kilinç, E. Karabulut, and T. F. Tözüm, "Analysis of cathepsin-K activity at tooth and dental implant sites and the potential of this enzyme in reflecting alveolar bone loss," Journal of Periodontology, vol. 83, no. 4, pp. 498-505, 2012.

[108] P. Gajendran, H. Parthasarathy, and A. Tadepalli, "Comparative evaluation of cathepsin $\mathrm{K}$ levels in gingival crevicular fluid among smoking and nonsmoking patients with chronic periodontitis," Indian Journal of Dental Research, vol. 29, no. 5, pp. 588-593, 2018.

[109] A. Beklen, A. Al-Samadi, and Y. Konttinen, "Expression of cathepsin $\mathrm{K}$ in periodontitis and in gingival fibroblasts," Oral Diseases, vol. 21, no. 2, pp. 163-169, 2015.

[110] M. Asagiri, T. Hirai, T. Kunigami et al., "Cathepsin K-dependent toll-like receptor 9 signaling revealed in experimental arthritis," Science, vol. 319, no. 5863, pp. 624-627, 2008.

[111] D. De Nardo, "Toll-like receptors: activation, signalling and transcriptional modulation," Cytokine, vol. 74, no. 2, pp. 181-189, 2015.

[112] K. Hayashi, M. Taura, and A. Iwasaki, "The interaction between IKKalpha and LC3 promotes type I interferon production through the TLR9-containing LAPosome," Science Signaling, vol. 11, no. 528, 2018.

[113] X. Li, Y. Cen, Y. Cai et al., "TLR9-ERK-mTOR signaling is critical for autophagic cell death induced by CpG oligodeoxynucleotide 107 combined with irradiation in glioma cells," Scientific Reports, vol. 6, no. 1, p. 27104, 2016.

[114] C.-S. Shi and J. H. Kehrl, "MyD88 and trif target beclin 1 to trigger autophagy in macrophages," Journal of Biological Chemistry, vol. 283, no. 48, pp. 33175-33182, 2008.

[115] A. Criollo, L. Senovilla, H. Authier et al., "The IKK complex contributes to the induction of autophagy," The EMBO Journal, vol. 29, no. 3, pp. 619-631, 2010.

[116] W. Pan, W. Yin, L. Yang et al., "Inhibition of Ctsk alleviates periodontitis and comorbid rheumatoid arthritis via downregulation of the TLR9 signalling pathway," Journal of Clinical Periodontology, vol. 46, no. 3, pp. 286-296, 2019. 